\title{
The Research on Calibration for Trigger Delay of Pulse Pattern Generator
}

\author{
Weihong Gu, Yang Yang, Jiajia Liu, Yongbin Bai \\ China Satellite Maritime Tracking and Control Department, Jiangyin 214431, China
}

Keywords: pulse pattern generator, trigger delay, system inherent delay.

\begin{abstract}
In this paper, the detection method of pulse delay generator 81134A triggering delay parameter is studied. Based on the different types of triggering delay output and the technical index, different measurement methods based on sampling oscilloscope, digital broadband oscilloscope and counter are designed. The system inherent delay, micro-delay measurement methods, and how to improve the measurement of the maximum measurement capacity and reading resolution of the measurement method.
\end{abstract}

\section{Introduction}

Pulse signal generator with frequency bandwidth, pulse output rising edge is extremely fast, is the modern scientific experiments, radar communication system testing and control of indispensable equipment, can be used for electronic measuring instruments and a variety of general, special instrument related pulse, Rise time, counting and other indicators of testing and testing.

JJG490-2002 "pulse signal generator verification procedures" is the latest state on the pulse signal generator verification procedures, pulse signal generator test items in the trigger delay and trigger (two) delay (hereinafter referred to as delay) Is the first test of the pulse signal generator, the subsequent verification and inspection of the use of the inspection items. As the technical indicators of different types of pulse signal generator is different, according to the test procedures to carry out the pulse signal generator verification and construction evaluation found that, for different parts, the program does not have clear different measurement standards of the use of occasions and measurement methods. In this paper, the measurement method and key technology of pulse signal generator triggering delay parameters are studied, and the measurement methods of different types of trigger delay parameters are designed, and the method of assessing the uncertainty of measurement results is given.

\section{Analysis of triggering delay test and test method}

JJG490-2002 "pulse signal generator verification procedures", the pulse signal generator trigger delay, trigger (or dual) delay of the verification method has been clear requirements, trigger delay test method sub-counter measurement and oscilloscope measurement Method, as shown in Figure 1 and Figure 2, a detailed description of the test method see the test procedures, do not repeat here. Refer to Section 3.5 for the metrological performance requirements for delay measurements given in the test procedure; see Section 5.2.2 for indicators for standard equipment requirements for verification.

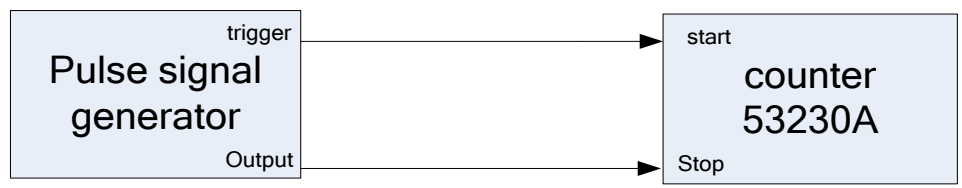

Figure 1. The counter measures the trigger delay 


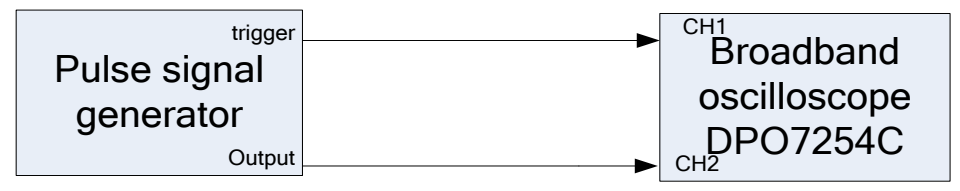

Figure 2. Broadband oscilloscope measures trigger delay

According to this test procedure, it is found that the trigger delay time range is $-5 \mathrm{~ns} \sim+230 \mathrm{~ns}$ and the delay resolution is $1 \mathrm{ps}$, because the technical index of the tested device is higher than that of the pulse code generator $81134 \mathrm{~A}$. The maximum allowable error is: $\pm 20 \mathrm{ps}$, technical indicators beyond the scope of the application, the simple use of the procedures provided by the verification method is difficult to normal measurement equipment error. Reference to the original Agilent 81134A performance test manual, the trigger delay parameter measurement using the standard is a digital broadband oscilloscope 54120T (bandwidth above $12.4 \mathrm{GHz}$ ), requiring the measured delay points of $1 \mathrm{~ns}, 1.5 \mathrm{~ns}$ and $2 \mathrm{~ns}$, the manual that the big Of the delay signal does not require verification, and there is no proposed to use the counter to measure the trigger delay parameters.

In this paper, the digital broadband oscilloscope DPO7254C (bandwidth $2.5 \mathrm{GHz}$, time base $2.5 \times$ $10^{-5}$ ), sampling oscilloscope 86100D (30GHz option 86112A-HBW) and counter 53230A (technical indicators $1.0 \times 10^{-8}$ ), respectively, 81134A trigger extension Time measurement test, the key technology involved in the triggering delay test is studied, and the triggering delay measurement range and measurement capability based on the oscilloscope and counter measurement standard are analyzed. Different measurement of the different trigger delay parameters of 81134A output is put forward.

\section{Research on Key Technology of Triggering Delay Test}

\subsection{Correctly set the correspondence between the pulse period and the trigger delay}

According to the conventional verification method, the smaller delay $(<3.5 \mathrm{~ns})$ is measured using a sampling oscilloscope or a digital broadband oscilloscope, and a delay of more than 3.5ns is using a digital broadband oscilloscope or counter. Using the counter to measure the delay, requiring the measured pulse period must be greater than the trigger delay or time interval, the actual measurement found, must also meet the pulse cycle is greater than the measured pulse signal generator system inherent delay $\mathrm{t}_{0}$, otherwise, the counter will give Error reading. Therefore, in the delay measurement, the first need to estimate or measure the measured system inherent delay $t_{0}$, and correctly set the pulse signal cycle is greater than the inherent delay.

The maximum capacity of the counter delay measurement should be less than the maximum cycle value of the pulse signal generator, and the actual capacity of the counter delay measurement will be smaller, which is related to the ability of the counter to automatically interpret the rising edge of the stop pulse. Likewise, the minimum delay of the measurement delay of the counter is related to the time interval measurement range of the counter and the inherent delay of the system. Using the oscilloscope to measure the delay, due to the use of manual readings, the pulse period is not necessarily greater than the inherent delay.

\subsection{System inherent delay to measurement method}

\subsubsection{The sampling oscilloscope measures the inherent delay}

In this procedure, the delay measurement requires the use of dedicated dual symmetrical delay cable to connect the pulse signal generator synchronous trigger output and delay output, the purpose is to reduce the test cable length and performance caused by different trigger signal and output pulse the difference between the delays. When the sampling oscilloscope $86100 \mathrm{D}$ is used to measure the delay, the 81134A triggering signal output terminal needs to pass the power divider in two ways to the trigger input of the sampling oscilloscope, and the other way to the sampling oscilloscope $\mathrm{CH} 1$, the delay output directly to the sampling oscilloscope $\mathrm{CH} 2$, as shown in Figure 3, there is no need to use a dedicated dual symmetrical delay cable, but must meet the bandwidth requirements of the cable, 
cable length and other factors affected by the delay change can be used as the system inherent delay $\mathrm{t}_{0}$ to eliminate.

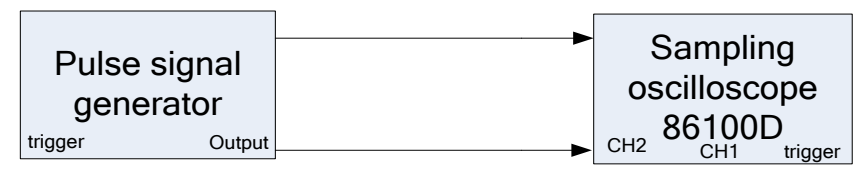

Figure 3. The sampling oscilloscope measures the trigger delay

\subsubsection{The counter measures the inherent delay}

Using the counter measurement method can not complete the 81134A full range of time delay measurement. Through the actual measurement found that the system inherent delay $t_{0}$ with the set output pulse cycle varies. For example, the output pulse period $\mathrm{T}$ is $20 \mathrm{~ns}$, the counter $53230 \mathrm{~A}$ measured the system inherent delay $\mathrm{t}_{0}$ is $13.51 \mathrm{~ns}$; output pulse period $\mathrm{T}$ is $50 \mathrm{~ns}$, the counter measured the inherent delay $\mathrm{t}_{0}$ is $33.54 \mathrm{~ns}$; therefore, in the measurement of each delay Value must be given at the same time the system of the system inherent delay $t_{0}$, the calculated delay value can be compared with the delay nominal value.

Using the counter 53230A to measure the 81134A delay also found that when the system inherent delay value $t_{0}$ plus the set delay value is close to or greater than the set output pulse period $\mathrm{T}$, the counter will give an error reading when measuring the time interval or delay Or far away from the normal value), therefore, in the delay measurement, the pulse cycle must be set large enough, of course, 81134A maximum cycle can only be 66ns, deduct the system inherent delay value $\mathrm{t}_{0}$, the counter can measure the maximum delay value, So the counter $53230 \mathrm{~A}$ can measure the maximum delay value of $81134 \mathrm{~A}$ is about 32ns. Counter 53230A measurement 81134A delay measurement results in Table 1.

Table 1. Counter 53230A measures the 81134A delay measurement results

\begin{tabular}{c|c|c|c}
\hline Pulse period $\mathrm{T}$ & Delayed nominal value & Delay reading & Inherent delay $\mathrm{t}_{0}$ \\
\hline 20ns & 0 & $13.51 \mathrm{~ns}$ & $13.51 \mathrm{~ns}$ \\
\hline $50 \mathrm{~ns}$ & 0 & $33.54 \mathrm{~ns}$ & $33.54 \mathrm{~ns}$ \\
\hline $50 \mathrm{~ns}$ & $10 \mathrm{~ns}$ & $43.53 \mathrm{~ns}$ & $33.54 \mathrm{~ns}$ \\
\hline $50 \mathrm{~ns}$ & $17 \mathrm{~ns}$ & $3.53 \mathrm{~ns}$ & $33.54 \mathrm{~ns}$ \\
\hline
\end{tabular}

\subsubsection{Broadband oscilloscope measures inherent delay}

Broadband oscilloscope measurement delay, you can use the cursor to manually measure the delay, you can not consider the pulse cycle must be greater than the delay limit, so the manufacturer provided 81134A performance test manual, the delay measurement does not use the counter, but the use of broadband Oscilloscope. Broadband oscilloscope 7254C measurement 81134A delay measurement results in Table 2.

Table 2. Oscilloscope DPO7254C measures 81134A delay measurement results

\begin{tabular}{c|c|c|c}
\hline Pulse period $\mathrm{T}$ & Delayed nominal value & Delay reading & Inherent delay $\mathrm{t}_{0}$ \\
\hline 20ns & 0 & $12.025 \mathrm{~ns}$ & $12.025 \mathrm{~ns}$ \\
\hline $20 \mathrm{~ns}$ & $20 \mathrm{~ns}$ & $32.05 \mathrm{~ns}$ & $12.025 \mathrm{~ns}$ \\
\hline $50 \mathrm{~ns}$ & 0 & $32.05 \mathrm{~ns}$ & $32.05 \mathrm{~ns}$ \\
\hline 50ns & $15 \mathrm{~ns}$ & $47.05 \mathrm{~ns}$ & $32.05 \mathrm{~ns}$ \\
\hline
\end{tabular}

\subsection{The Method of Measuring the Accuracy of Readout by Broadband Oscilloscope}

How to improve the reading accuracy of broadband oscilloscope delay measurement, especially the large delay measurement $(\geq 100 \mathrm{~ns})$ is one of the problems studied in this paper. The oscilloscope screen displays both the trigger pulse and the rising edge of the delayed output pulse. The large delay measurement must require that the oscilloscope set a large time base and that the introduction of the oscilloscope reading error will also increase.

The DPO7254C time interval measurement index is: the average sampling mode of the oscilloscope (average number of times $>100$ times), the bandwidth is limited to full bandwidth, the maximum allowable error is $\pm\left(2.5 \times 10^{-6} \times \mid\right.$ reading $\left.\mid+4 \mathrm{ps}\right)$, the measurement error is directly related 
to the reading value. By setting the oscilloscope, use the oscilloscope $\Delta t$ cursor measurement function to manually measure the delay, keep the oscilloscope cursor $\mathrm{X}_{1}$ accurately aligned 81134A trigger output pulse rising edge $50 \%$, and gradually increase the delay output value, and set the oscilloscope horizontal time base gear, Output pulse rising edge, keep the rising edge displayed in the screen, the same way the cursor $\mathrm{X}_{2}$ accurately aligned output pulse rising edge $50 \%$, and finally read $\Delta t$ value. By setting the oscilloscope in this way, you can ensure that the cursor $X_{2}$ does not misalign the other rising edges when setting the large delay output. Oscilloscope measurement of large delay measurement results in Table 3.

Table 3. The oscilloscope measures large delay measurements

\begin{tabular}{c|c|c|c|c|c}
\hline $\begin{array}{c}\text { Pulse } \\
\text { period T }\end{array}$ & $\begin{array}{c}\text { Delayed } \\
\text { nominal value }\end{array}$ & $\begin{array}{c}\text { Delay } \\
\text { reading }\end{array}$ & $\begin{array}{c}\text { Inherent } \\
\text { delay } \mathrm{t}_{0}\end{array}$ & $\begin{array}{c}\text { Delay } \\
\text { measurement }\end{array}$ & $\begin{array}{c}\text { Oscilloscope time } \\
\text { base }\end{array}$ \\
\hline $50 \mathrm{~ns}$ & 0 & $32.0 \mathrm{~ns}$ & $32.0 \mathrm{~ns}$ & 0 & \\
\hline $50 \mathrm{~ns}$ & $100 \mathrm{~ns}$ & $132.0 \mathrm{~ns}$ & $32.0 \mathrm{~ns}$ & $100.0 \mathrm{~ns}$ & $20 \mathrm{~ns} / \mathrm{div}$ \\
\hline $50 \mathrm{~ns}$ & $230 \mathrm{~ns}$ & $262.0 \mathrm{~ns}$ & 32.0 & $230.0 \mathrm{~ns}$ & $50 \mathrm{~ns} / \mathrm{div}$ \\
\hline
\end{tabular}

3.4 Analysis of Factors Affecting Measurement of Micro Delay in Sampling Oscilloscope

Using the sampling oscilloscope to measure the 81134A delay As shown in Figure 3, the measurement of small delay must take into account the measurement resolution and measurement accuracy and several other factors. 81134A output micro-delay (3.5ns below) measurement can be measured using 86100D, sampling oscilloscope minimum time base: 2ps / div, time interval measurement accuracy: $1 \mathrm{ps}+1.0 \% \Delta \mathrm{t}$ or $8 \mathrm{ps}$, whichever is smaller $(0=1,2, \ldots 18)$, the time interval measurement accuracy is: $8 \mathrm{ps}+0.1 \% \Delta \mathrm{t}$. When the delay value is set across $(28+4 \mathrm{~N}) \mathrm{ns} \pm 100 \mathrm{ps}$, As the inherent delay of the $86100 \mathrm{D}$ itself is $24 \mathrm{~ns}$, when the $81134 \mathrm{~A}$ output delay is greater than $4 \mathrm{~ns}$, $\Delta \mathrm{t} \geq 28 \mathrm{~ns}$, the time interval measurement accuracy: $8 \mathrm{ps}+0.1 \% \Delta \mathrm{t}$, in the measurement results uncertainty assessment, we must pay attention to these jump points On the uncertainty of the measurement results.

Sampling oscilloscope measurement delay method is on the same screen at the same time display the rising edge of the trigger pulse and the measured pulse signal rising edge, the use of $\Delta \mathrm{t}$ cursor measurement function read delay value. The system inherent delay is large, about 35ns, this time, the sampling time of the oscilloscope is $5 \mathrm{~ns} / \mathrm{div}$, due to the limitations of the oscilloscope readings, artificial naked eye is difficult to accurately distinguish the small delay, time interval reading resolution of 50ns / $4096=12 \mathrm{ps}>62.5 \mathrm{fs}$, take 12ps, can not play the advantages of the oscilloscope. Horizontal time base expanded to 10ps / div, then the time interval reading resolution of 100ps / 4096 $=0.024 \mathrm{ps}<62.5 \mathrm{fs}$, take 62.5fs (86100D time interval resolution: $\leq$ (screen diameter) / (record length) or $62.5 \mathrm{fs}$, Both take the big).

\section{Summary}

In this paper, through the use of sampling oscilloscope, broadband oscilloscope and the counter pulse code generator 81134A trigger delay parameter verification problems encountered and key technologies to carry out research, for different types of trigger delay parameters, designed a variety of solutions, It is helpful to carry out the verification of the trigger delay parameter of the pulse pattern generator 81134A according to the existing pulse signal generator verification procedure.

\section{References}

[1] Agilent 81134A Performance Verification

[2] Du Jianguo, Zhao Ziwen, etc. JJG490-2002 "pulse signal generator verification procedures" [M]. Beijing: China Metrology Publishing House, 2002.11.

[3] Liu Mingliang, Lu Fumin, Zhu Jiangmiao, Yu Yuehua. "Modern pulse measurement" [M]. Beijing: Science Press, 2010.05.

[4] Tektronix DPO70000C Technical Specifications Test Reference Manual 